\title{
MANUEL TOUSSAINT Y LA PINTURA COLONIAL
}

\section{$\mathbf{P} \mathbf{O} \mathbf{R}$}

\section{E L IS A VARGAS LUGO}

$\mathrm{F}^{\mathrm{L}}$ estudio de la pintura colonial fue el aspecto del arte novohispano, que interesó sobre todos, de manera especial y personal, a don Manuel Toussaint. En todas sus obras, sus conferencias y sus lecciones de arte, el maestro nos demostró los amplios conocimientos que tenía de las artes plásticas en general, pero si nos preguntáramos cuál fue su preferencia dentro de este campo, no dudaríamos en decir que la pintura, cuyo estudio fue para él, algo así como su especialidad en la profesión de investigador del arte mexicano.

Por el sentido de su obra, por su lenguaje, por la forma erudita, objetiva y clara de abordar los temas, por su método histórico - herencia legítima y postrera del espíritu positivista que tanto arraigo tuvo en México-, don Manuel Toussaint pertenece a un grupo de escritores post-revolucionarios que recogieron en sus obras la continuación de la historia del arte y de la historia de la pintura en particular, iniciada por el doctor Lucio a mediados del siglo pasado, e interrumpida durante años por causa de las vicisitudes políticas que sufrió el país. Este grupo de intelectuales cuyos frutos comenzaron a publicarse hacia 1920 se inicia con Francisco Díez Barroso, quien publicó en 1921 El Arte en Nueva España, con una parte dedicada a la pintura; luego encontramos, en 1922, el libro de Manuel Romero de Terreros Historia Sintética del Arte Colonial de México, también con un capítulo sobre pintura; en 1923 la obra de Francisco Pérez Salazar: Algunos datos sobre la Pintura en Puebla en la Epoca Colonial, y uno o dos años después, una segunda edición 
aumentada ; Datos para la Historia de la Pintura en Puebla; algunos años más tarde, en 1927, el interesantísimo estudio de José Juan Tablada, titulado La Historia del Arte en México; en 1934, La Pintura en México Durante el siglo XVI, del propio Manuel Toussaint; de Agustin Velázquez Chávez, en 1938, Tres Siglos de Pintura Colonial Mexicana, y finalmente, también del maestro Toussaint, su gran libro sobre Arte Colonial en México, impreso en 1948 en donde el autor recoge todos los datos conocidos hasta entonces, más las informaciones obtenidas por sus investigaciones particulares, of reciéndonos asi, en los capítulos, que en este libro dedica a la pintura, el panorama más completo que se ha escrito sobre la historia de este arte.

La lectura de tales obras nos proporciona una imagen fiel del estado casi incipiente en que se encontraban los estudios del arte mexicano hasta hace muy pocos años y esto precisamente nos hace valorar en su justo mérito tales aportaciones, que de manera tan considerable han contribuído al conocimiento de esos temas. De las informaciones alcanzadas por Couto y Revilla y otros autores de segunda importancia, durante los últimos años del siglo pasado, poco nuevo se agregó en obras como la de Baxter, de 1901, por ejemplo, que como bien dijo don Manuel Toussaint, en la mayor parte de su capítulo sobre pintura, Baxter repitió a Couto a Lucio y a Revilla. No sucedió asi con los trabajos del grupo post-revolucionario que mencionamos, quienes, con otros ojos, desligados ya del academismo que padecieron tan agudamente autores de la talla de Couto, abrieron los nuevos caminos de la investigación histórico-artistica. De tales autores y obras mencionados arriba, no es propio hacer aquí un análisis particular, pero sí es necesario decir que cada uno contribuyó a la historia de la pintura colonial con valiosos informes, fruto de sus íntimas preocupaciones, y que - lo más importante para la finalidad que nos acupa-, estos escritores tienen varios rasgos comunes, que los caracterizan y relacionan entre si y por los cuales consideramos que forman un grupo, coronado por la figura de Manuel Toussaint.

Desde luego no hará falta explicar con amplitud, por qué dichas características de que hablamos, son producto de las circunstancias históricas y culturales que vivieron estos escritores, pero deseamos mencionarlas porque precisamente, en la superación de tales circunstancias estriba el éxito intelectual de Manuel Toussaint.

Se destacan desde luego, a través de la lectura cuidadosa de las obras que estamos considerando, tres rasgos muy importantes: la afición de los autores por el estudio del arte, en el sentido de que todos empe- 
zaron sus trabajos, por verdadero amor al arte, fuera de toda institución cultural, fuera de todo método científico y en la mayoría de los casos al margen de las actividades profesionales; el carácter fundamentalmente erudito, más que crítico de las obras, cosa que ya mencionábamos at principio de este párrafo, y una tendencia nacionalista, en las obras más importantes de este conjunto, que se pone de manifiesto en las páginas dedicadas al estudio de la pintura indígena o a la decisiva contribución de los pintores indigenas, en el campo, ya de la pintura europea.

Si bien es cierto que dichas caracteristicas, como podria demostrarse: de un análisis más profundo, como decíamos, son producto de las circunstancias históricas, no quiere esto decir que todas las obras tengan por lo tanto, igual mérito o sean de la misma importancia. El mérito y la importancia de cada una de ellas radica en el matiz que dentro de esas bases comunes, le dio cada autor. Así, nos resulta la obra de Toussaint la más valiosa, la más completa e interesante, sobre historia de la pintura. colonial. Porque Toussaint no permaneció siendo, como cuando escribió sus primeros artículos sobre arte, un aficionado, sino que cayendo en cuenta de la urgente necesidad que habia de que dichos trabajos se hicieran con método científico y carácter académico, fundó el Laboratorio de Arte en 1934, que años más tarde se transformó en este Instituto de Investigaciones Estéticas, encauzando de esta manera, definitivamente, los estudios del arte en México porque en sus obras la erudición no es su meta final. El supo escapar a ese pecado de moda todavía en los años que él inició sus escritos $-y$ aún hoy en dia--, para dar a estos un: sentido más trascendente, y por lo que toca al aspecto nacionalista de su obra, porque si bien ya José Juan Tablada habia hecho justicia en su Historia del Arte en México, al arte indigena, concediéndole igual categoría que al arte europeo, en cuanto tal, nuestro autor dio los pasos que seguían a este primero, dando a conocer diferentes aspectos de la participación de los artistas indigenas en la propagación de la pintura europea, como explicaremos adelante.

La obra escrita por don Manuel Toussaint sobre pintura colonial recoge, fundamentalmente las dos publicaciones mencionadas ya, que son muy conocidas y en su póstuma obra, inédita aún, que esperamos saldrá a luz en breve, además de sinnúmero de articulos sobre distintos temas pictóricos y pintores en particular, publicados en diferentes revistas y periódicos, que no consideraremos en este trabajo. Las publicaciones a que nos referimos, son: La pintura en México durante el siglo XVI, publicada en 1936 y los capítulos correspondientes a la materia que nos 
ocupa, en su Arte Colonial en México, de 1948. Por medio de estos textos trataremos de presentar los méritos, ya anunciados, de la obra de Toussaint por lo que se refiere al estudio de la pintura colonial, las novedades que presenta respecto de los demás autores y las grandes aportaciones que logró hacernos su incansable espíritu de investigador.

Nueva visión del problema.

En su libro sobre La pintura en México durante el siglo XVI, primera publicación de conjunto escrita por el maestro Toussaint, aparecen ya todas las innovaciones en cuanto al planteamiento empleado para el 'studio de la pintura colonial, mismo planteamiento que volveremos a cncontrar en su segunda publicación titulada Arte Colonial en México, si bien ampliando y rectificando en algunos puntos, según lo exigieron sus mayores conocimientos sobre la materia.

En esta obra Toussaint divide en quince capitulos los temas pictóticos de la primera centuria novohispana, en forma verdaderamente sistemática, por primera vez desde que se escribía historia del arte. La sola cnunciación de esos capítulos -que a continuación hacemos-, muestra d gran adelanto de sus conocimientos sobre pintura colonial, respecto a las últimas publicaciones hechas con anterioridad a esta suya. Dichos capitulos son los siguientes: I. La pintura en México durante el siglo XVI (breve introducción); II. La pintura que existía en México a la llcgada de los españoles; III. La pintura que traían los europeos; IV. Causas que motivaron la aparición de la pintura europea en México; V. Decoración de templos y conventos en el siglo XVI; VI. Conventos franciscanos; VII. Conventos agustinianos; VIII. Conventos dominicanos; IX. Códices post-cortesianos; X. El primer pintor europeo. El problema do Rodrigo de Cifuentes; XI. La pintura en México a mediados del siglor XVI; XII. El primer gran pintor de la época colonial, Simón Pereyns; XIIL. Artistas que forman al grupo de Simón Percyns; XIV. Conclusion y XV. Bibliografía.

Para cualquier estudioso del arte mexicano, conocedor de las principales obras que, para el efecto, forman nuestra bibliografía, será fácil darse cuenta de la novedad e importancia de este planteamiento hecho pór don Manuel Toussaint para el estudio de la pintura novohispana del siglo XVI. Este planteamiento es en sí, un gran paso según la manera de 
considerar las obras pictóricas y los métodos empleados hasta entonces. Todas las obras anteriores presentan panoramas cronológicos, pero globales, se puede decir, como Revilla, ${ }^{1}$ Couto, $^{2}$ o Tablada, ${ }^{8}$ por ejemplo, que nos hablan desde Pereyns hasta el academismo pero sin diferenciar con claridad las etapas y escuelas pictóricas y las diferentes causas que forman la historia de nuestra pintura. Manuel Toussaint al plantearse una forma sistemática, más amplia, más ambiciosa y sobre todo más lógica, del fenómeno histórico-pictórico - ya no sólo artístico-, enriqueció notablemente el panorama de este fenómeno cultural y dejó establecidas las bases para futuras investigaciones. Cada uno de sus capitulos tiene irrefutablemente, un sentido histórico y artístico. Sólo el mismo Toussaint ha superado este programa ampliándolo en su libro posterior. Toda aportación futura tendrá que basarse en él, porque han quedado señalados los puntos claves de la trayectoria que siguió la pintura en el siglo xvi. Por primera vez, los datos conocidos hasta entonces, las obras realizadas por otros autores y las investigaciones personales del maestro Toussaint, sirvieron para establecer en una síntesis - como él mismo lo dice- ${ }^{4}$ el esquema sobre el cual habrá de realizarse el estudio de dicho arte, Muchos de sus capitulos podrian modificarse por su contenido, otros podrian desaparecer, pero la mayor parte son enunciados definitivos, constitutivos - históricamente hablando- de tal desarrollo artístico. Así, por ejemplo, nadie, al hablar de pintura mural podrá dejar de establecer la distinción que existe entre los frescos de los conventos dominicanos, agustinianos o franciscanos. Estas categorías distintas entre si por razones históricas y artísticas quedaron descubiertas por la pluma de Manuel Toussaint. (Capítulos v, vi, viI y virI de la obra mencionada.)

Su segunda publicación, Arte Colonial en México, tiene un carácter distinto a la primera que acabamos de considerar, porque no trata solamente sobre pintura, sino sobre las artes plásticas en general. Publicada

1 Manuel G. Revilla, El Arte en México en la época antigıa y durant el gobierno virreinal. México, 1893.

2 Bernardo Couto, Diálogos sobre la Historia de la pintura en Mexrico. México, 1947.

3 José Iuan Tablada, $O p$. cit.

4 "Con los cortos elementos que existen, investigaciones en archivos, exploraciones de monumentos, etc., me propongo realizar un pequeño resumen..." Manuel Toussaint, La pintura en México en el siglo XVI, México, 1936, p. 5. 
en 1948, a más de diez años de distancia de la anterior, revela inmediatamente el tiempo transcurrido, en el mayor acopio de datos y en la presentación de una obra de conjunto. En ella el maestro modificó y amplió varios puntos claves, para el estudio de la pintura y de las artes plásticas en general, como veremos a través del análisis del indice de dicho libro. Para empezar, el panorama cronológico abarca toda la época colonial, hasta el advenimiento del neoclasicismo, dividido para su estudio en veinte y cinco capitulos, pero con la novedad de que los subdivide, primero, en función de los procesos históricos acaecilos en la Nueva España y luego en función de las escuelas artisticas que florecieron durante dichas etapas históricas. Haciendo un esquema del indice, para lograr mayor claridad en nuestras afirmaciones, se obtendria el siguiente cuadro:

\begin{tabular}{|c|c|c|}
\hline División cronológica & Proceso histórico & Formas artísticas \\
\hline $\begin{array}{c}\text { Primera parte } \\
(1519-1550)\end{array}$ & $\begin{array}{l}\text { El Arte en la Nueza } \\
\text { España en tiempos } \\
\text { de la Conquista. }\end{array}$ & $\begin{array}{c}\text { La Edad Media en } \\
\text { México. }\end{array}$ \\
\hline $\begin{array}{c}\text { Segunda parte } \\
(1550-1630)\end{array}$ & La Colonización. & $\begin{array}{c}\text { El Renacimiento en } \\
\text { México. }\end{array}$ \\
\hline $\begin{array}{c}\text { Tercera pcrte } \\
(1630-1730)\end{array}$ & $\begin{array}{c}\text { El Arte cn la Nueza } \\
\text { España durante la } \\
\text { formación de la } \mathrm{Na-} \\
\text { cionalidad. }\end{array}$ & El estilo Barroco. \\
\hline $\begin{array}{l}\text { Cuarta parte } \\
(1730-1781)\end{array}$ & Orgullo y riqueza & $\begin{array}{l}\text { Apogeo del Arte } \\
\text { Barroco en México. }\end{array}$ \\
\hline $\begin{array}{l}\text { Quinta parte } \\
(1781-1821)\end{array}$ & $\begin{array}{c}\text { Ideas de realización } \\
\text { de la Independencia } \\
\text { de México. }\end{array}$ & El Arte Neoclásico. \\
\hline
\end{tabular}

Este valioso índice cronológico histórico-artístico, ofrece al lector un magnífico panorama del desarrollo de las artes plásticas mexicanas durante la colonia. La base cronológica necesaria, pero no siempre suficiente, está utilizada con propiedad, en el justo sitio que le corresponde, sirviendo sólo de gran minutero para saber la hora exacta en que tuvo lugar tal o cual hecho histórico o artístico. La intima relación que existe entre las formas artísticas y sus determinantes históricas, 
está puesta de manifiesto en la correspondencia establecida entre los títulos y subtítulos. Así, por ejemplo, la Cuarta Parte qu'e abarca de 1730 a 1781, titulada Orgullo y Riqueza, tiene por subtítulo Apogeo del Arte Barroco. La relación es clara. Hacia la segunda mitad del siglo xvirI la riqueza novohispana era abundante y oropelesca. Los criollos pudieron ornamentar sus iglesias con ricos altares y portadas suntuosas del más exuberante Arte Barroco. No cabe duda que la riqueza de la colonia fue una de las causas directas en el desarrollo de las artes plásticas de esa época, aunque desde luego no fue la única. Otros motivos concurrieron a determinarlo que no corresponde analizar en este momento. Lo que hoy nos interesa es hacer notar que el valor de este segundo tratado sobre la pintura colonial, es el hecho de estudiar su desarrollo en el Virreinato no sólo por siglos, sino por formas artísticas y períodos históricos. Esto fue otro paso definitivo en la carrera de nuestro investigador. Paso obligado y bien realizado después de su primera publicación. Es curioso darse cuenta de cómo autores de la talla de Couto debido a su ultra-academismo, pero podría ser por otra razón y para el caso es lo mismo, no usó para nada la palabra Barroco, jen un libro sobre pintura colonial! Ni tampoco más tarde José Juan Tablada cuyo libro está lleno de brillantes ideas -algunas todavia preocupaciones de nuestros dias- pudo ofrecernos un panorama más diferenciado de nuestra pintura colonial. Pero no se trata aquí de menospreciar a nadie, sí de hacer resaltar, un poco por contraste, el valor que tienen las obras de Manuel Toussaint. Así pues con su segundo libro nos adentramos hasta la pintura de los siglos xvir, xviri $y$ xIx, pero, como hemos visto, no sólo bajo el ritmo del tiempo, sino también con espíritu histórico-artístico propiamente dichos.

\section{El fundamento histórico.}

Para completar como se debe la idea que queremos dar sobre la obra de Toussaint, falta hacer hincapié en su actitud de historiador, que es donde radica la parte realmente trascendental de sus estudios. Es decir, que gracias a ese sentido histórico con que analizó la pintura y el arte colonial en general, trató de fundamęntar históricamente - como hemos dicho- estos fenómenos artísticos, logrando, en su intento, darles su explicación humana $y$ por ende trascendente, sin la cual la 
erudición artística nos parece cosa inútil y muerta. Las obras de otros autores, unas anteriores a las de Toussaint y otras contemporáneas a él no intentaron dicha fundamentación histórica del arte. Por esto scguramente han perdido interés y valor ante nuestros ojos. En muchas de ellas - no todas desde luego- echamos de menos la explicación del nacimiento y desarrollo de la pintura y sus diferentes etapas y matices. Unos más otros menos, repiten el papel de la Iglesia en los principios de la aparición de la pintura en la Nueva España, pero rara vez tratan de ir más allá, para preguntarse seriamente sobre los "por qué", abundantísimos, del fenómeno pictórico novohispánico. Antes de Toussaint ningún autor explica, por ejemplo, por qué aparecen las características del arte medieval en la Nueva España. Apenas se mencionan un tanto confusas, las escuelas pictóricas europeas que florecieron aqui. $\mathrm{Ni}$ nadie antes explicó el fenómeno de la pintura mural que ornamenta tan ricamente los monasterios del siglo xvi. Nuestro autor, sin embargo abordó estos y otros temas igualmente importantes respondiendo a las interrogaciones por medio de la historia. Pero dejemos la palabra en este momento al propio Manuel Toussaint, que será más elocuente y más vivo que cuanto podamos decir al respecto:

Al hablarnos de los comienzos de la pintura en el siglo xvi, acli:rando, por primera vez, la forma en que pasó la pintura europea a la Nueva España para explicar ciertos caracteres artísticos nos dice, históricamente:

"En el momento de verificarse la conquista de México, el occidente de Europa acababa de despertar a una nueva vida: el Renacimiento. l.a Edad Media, austera, de severidad artística rayana en ascetismo, cuyas ideas artísticas se encontraban supeditadas en todo a la iglesia, porque la iglesia había sido el baluarte de la libertad y de la ciencia, sc iba transformando poco a poco en una existencia más mundana, más fastuosa, a la vez que la sociedad pugnaba por adquirir los derechos individuales en contra del feudalismo cada vez más anacrónico y decalente. $\mathrm{El}$ poder regio tenía que establecerse a base de concesiones de más libertades a los súbditos, a fin de que éstos apoyasen al rey contra sus primitivos señores. El auge del comercio originó los grandes capitales y los ricos ambicionaron poseer obras artísticas para adorno de sus mansiones y ostentación de su nueva aristocracia.

Pero en España presidia aún el espíritu medieval; además, la colonia, a seis meses de distancia, se encontraba mucho más lejana con respecto al arte de moda, asi que las primeras obras de arte que llegan 
a México son todavía de pleno siglo $x v, y$ pertenecen aún en parte a esa categoría deliciosa de pinturas que se conocen con el nombre de primitivas." $"$

Ya dentro del siglo xvil hablando sobre la aparente incompatibilidad de las pinturas atribuídas a Sebastián López de Arteaga, "La incredulidad de Santo Tomás" y "Los Desposorios", cosa que a la mayor parte de las personas les parecía ilógica, dada la gran diferencia de colorido y composición que existe entre ambos cuadros, Toussaint dice sabiamente :

"Si nos atenemos a la realidad histórica, podemos explicar cómo ambas obras pueden haber sido del mismo pintor. Desde luego advertimos que críticos distinguidos de la pintura europea, no encuentran diferencias tan radicales entre estos dos cuadros, como puede existir en otras pinturas: el mismo Zurbarán presenta diferencias en sus 'maneras', acaso más acentuadas. Yo creo que Arteaga al llegar a México encuentra que la Colonia se delecta profundamente en el arte pictórico que habia implantado Echave y habían seguido fielmente sus discípulos. Arte suave, de marcado italianismo en el brillo de los colores, en la suavidad de toque, en la ausencia de problemas dramáticos. El pintor, que necesita vivir, puesto que no existe todavía en una época en que el artista impone su voluntad al público, sino que es aún un ártesano que tiene que atender los pedidos que se le hacen con las indicaciones, muchas veces terribles, de los clientes, no va a dejar de pintar porque las obras que realiza no sean de su intimo gusto: pinta el Desposorio, el Retrato del señor Manzo y Zúñiga, el Arcangel San Miguel, acaso los Retratos de los Inquisidores, y quién sabe cuantos cuadros más que no conocemos. Cuando Arteaga logra el apoyo del Santo Oficio y puede pintar un cuadro en que no se le impone ninguna tendencia, desarrolla completamente su personalidad y su gusto y entonces produce una obra que se destaca de todas las demás y viene a ser como un jalón en la historia de la pintura mexicana. Así pasó con la Incredulidad de Santo Tomás." 6

Quedando así aclarado el sorprendente barroquismo de fuerte arraigo español con que López de Arteaga seguramente con gran asombro p. 9.

5 Manuel Toussaint, La pintura en México en el siglo XVI, México, 1936.

6 Manuel Toussaint, Arte Colonial en México, México, 1948. Cap. Iv, p. 155. 
de sus contemporáneos, realizó su preciosa pintura y otra más, conocida por nosotros: La Crucifixión.

Al acercarse a la gran etapa del arte barroco nexicano Toussaint nos explica en el capítulo $\mathrm{Ix},{ }^{7}$ de su Arte Colonial de México, algunas de las causas históricas que motivaron este arte en la colonia, al que - dicho sea de paso- él considera una faceta exponente del sentimiento de nacionalidad que por esos años empezaba a tomar forma entre los habitantes de la colonia:

"Asistimos en esta tercera época de nuestro recorrido al fenómeno más interesante que presenta la historia de México. Los primitivos conquistadores que más tarde se tornaron en colonos, han muerto: sus descendientes disfrutan de las enormes riquezas que ellos les habian legado; estos descendientes han nacido en México, se han casado con mujeres nacidas en el país y algunos aún con indias. Se va, pues, diferenciando el grupo étnico español que habita en la Nueva España del grupo original que le diera ser, de la metrópoli."

Enunciando así la diferenciación entre criollos y gachupines, nos dice más adelante:

"Pero es el estado social de la Nueva España en aquella época al que debemos dirigir nuestra atención para precisar la nueva modalidad de estilo que impregna al arte. Es una sociedad eminentemente aristocrática de aristocracia de dinero, que ostentaba como única nobleza, el descender de los conquistadores o el ejercitar las mayores obras pías o de caridad que se pudiera. Si en la época anterior los templos y conventos $y$ aún las mismas catedrales, fueron edificados en gran parte con el patrimonio de la corona y otros subsidios obligatorios, en esta época fueron los mismos potentados de la colonia los que contribuyen al auge intenso de la arquitectura religiosa. Enormes fortunas son destinadas por los mayorazgos o por los simples caballeros para constituir patronatos en templos y conventos. Se dijera que existe una especie de arreglo tácito entre estos hombres que llevan una vida a veces licenciosa, $y$ la Iglesia, que les of rece, por lo menos según ellos creen, la salvación de sus almas si conceden grandes donativos para edificar templos y conventos..." "Por lo que se refiere a los estilos artísticos, asistimos a una renovación total de criterio: no son ya las obras platerescas anteriores las que han de imperar en los templos: se va elaborando una nueva modalidad estilistica que consiste en tomar clementos de todas las formas

7 Ibid. Cap. Ix, pp. 191-192. 
anteriores y construir con ellas un nuevo estilo. Por lo demás, el mismo fenómeno había ocurrido en Europa: el nuevo estilo del Viejo y dei Nuevo Continente se llama barroco."

Con la transcripción de estos párrafos hemos querido presentar, aunque fuera a grandes rasgos, una idea del método seguido por Toussaint a lo largo de su libro en un intento de dar a conocer el trasfondo histórico de los fenómenos artísticos, tal como venimos indicando desde el principio de este estudio.

Otros aspectos interesantes de la obra de Toussaint son, dichos ya breve y someramente, el haber hecho luz sobre el estudio de la pintura mural, para lo cual no sólo investigó en los archivos en busca de datos, sino que viajó incansable, para obtener la impresión viva de las obras de arte; $y$ las valiosas rectificaciones hechas a varios autores, concretamente sobre pintores y obras pictóricas. Para darse una idea más amplia de este aspecto del trabajo realizado por Toussaint, basta con leer las introducciones y anotaciones a los libros, primero de Silvester Baxter Arquitectura Hispano Colonial en México ${ }^{8}$-que dedica un capitulo a la pintura-, y luego al de Bernardo Couto ${ }^{9}$ Diálogo sobre la Historia de la Pintura en México. Sobre todo en este último, encontramos rectificaciones tan valiosas, como las que siguen:

En la página 42, de dicha obra, refiriéndose al problema existente sobre la personalidad del primer pintor europeo en Nueva España Toussaint aclara: "Lo más probable es que el primer pintor que pasó a Nueva España haya sido Cristóbal de Quesada, que afirma que en 1540, lo envió don Antonio de Mendoza en la expedición de Francisco Vázquez de Coronado", rematando asi la ya desmentida suplerchería sobre el pintor Rodrigo de Cifuentes a quien se consideraba, como ya sabemos, el primer pintor novohispano. Más adelante, en las páginas 55 y 56 de la misma obra, nos aclara uno de los más enigmáticos problemas que se habian presentado dentro de la pintura colonial : el caso de los Echave. Toussaint fué el primero en llegar al conocimiento de que tales pintores no habían sido sólo dos, como por tanto tiempo se pensó, i sino nada menos que cuatro! La explicación documentada de este hallazgo es demasiado larga para transcribirla, pero el lector puede encontrarla en el libro citado. Basta con advertir la importancia de tal rectificación que como se supondrá fué de utilidad incalculable, ya que entre la vida y obra de los

8 Silvester Baxter, Arquitectura Hispano Colonial en México. México, 1901.

9 Bernardo Couto, Op. Cit. 
cuatro Echaves, transcurrió más o menos un siglo. Una confusión en un lapso de tiempo tan grande era un gran obstáculo para el conocimiento de la historia de la pintura, desaparecido éste gracias a las investigaciones de Manuel Toussaint, se ha logrado atribuir correctamente los distintos cuadros pintados por los Echaves a sus respectivos auténticos autores, y además se delucidaron ciertos problemas pictóricos y de anacronismo, que antes no era posible entender.

Como estos ejemplos podriamos mencionar muchísimos, en los cuales, uno a uno, se demuestra -a más del propio valor de la información en sí- la acuciosidad, la erudición, el sentido artístico e histórico del historiador del arte Manuel Toussaint, pero eso implicaría un trabajo exhaustivo y voluminoso que esta rápida consideración de su obra no pretende hacer. Con la próxima aparición de su obra inédita estos aspectos se enriquecerán enormemente. En ella se encontrará un gran acerbo de información sobre pintores de primera y segunda importancia, hasta ahora desconocidos. Estamos seguros de que esta nueva publicación completará de manera igualmente valiosa para la historia de la pintura los esfuerzos realizados en las dos obras tratadas aquí.

Hoy la historia del arte se comienza a hacer con otros ojos, tan nuevos como un día los tuvo Manuel Toussaint para poder proyectarlos hacia el futuro y dar a los estudios del arte la calidad que entonces se hacia necesaria. Pero precisamente esta nueva misión de las cosas es la que hoy nos ha llevado a considerar el valor de las obras del desaparecido investigador.

Para terminar diremos, repitiendo un poco, que la calidad de la obra de Toussaint, lo mejor, lo más valioso de ella, es que el autor, sin llegar a ser nunca historicista - cosa que por cierto, a veces se nos antojaría muchísimo- intuyó que el secreto para comprender el fenómeno artístico, es decir, para lograr la explicación de su razón de ser como forma de arte, estaba en el conocimiento de la historia que envuelve a tal o cual fenómeno artístico, y a ella acudió para resolver los problemas que se le presentaron, obteniendo los magníficos resultados que aquí hemos tratado de hacer sobresalir para hacer justicia a su labor intelectual. 